\title{
Utility of ranolazine in chronic stable angina patients
}

\author{
Pawan D Patel \\ Rohit R Arora \\ Department of Cardiology, Chicago \\ Medical School, North Chicago, IL, USA
}

\begin{abstract}
Chronic stable angina is a debilitating illness affecting at least 6.6 million US residents. Despite being optimally treated by pharmacotherapy and revascularization up to $26 \%$ of patients still experience angina. Diabetes mellitus is a common co-morbid condition in angina patients. Several new investigational medications are being tested for chronic angina. Advances in understanding of myocardial ischemia have prompted evaluation of a number of new antianginal strategies. In this review we discuss the utility of ranolazine, a recently approved novel antianginal agent and its efficacy in the diabetic patient population. In addition to its antianginal action in diabetic patients with chronic angina, ranolazine may have favorable effects on glycated hemoglobin levels.
\end{abstract}

Keywords: chronic stable angina, antianginal, ranolazine, diabetes mellitus, glycated hemoglobin

\section{Introduction}

Chronic stable angina is a debilitating illness affecting atleast 6.6 million US residents and approximately 400,000 new cases of stable angina occur each year (Gibbons et al 2003). Angina has an annual death rate of 1.6\%-3.2\% (Gibbons et al 2003). Furthermore, up to $26 \%$ of patients experience angina despite being treated by revascularization and pharmacology (Serruys et al 2001; Holubkov et al 2002).

Chronic stable angina is an insidious manifestation of coronary artery disease (CAD). In the US, 5\%-15\% of the 12 million patients with chronic angina have refractory angina. In order to improve the quality of life, the integrated approach to CAD should focus on cardiovascular events and prevention of anginal symptoms (Bhatt and Stone 2006).

According to National Cholesterol Education Program (NCEP) - Adult Treatment Panel (ATP) III guidelines, diabetes mellitus is a CAD risk equivalent (Adult Treatment Panel III 2002). Because diabetic patients have substantial morbidity due to $\mathrm{CAD}$, glycated hemoglobin (HbA1c) is an integral part of their CAD management. In a meta-analysis on 7,500 diabetic patients a one percentage point increase in $\mathrm{HbA} 1 \mathrm{c}$ was linked to an $18 \%$ increased risk of heart attack or stroke, and a $28 \%$ increase in the risk of peripheral vascular disease. Accordingly, someone with an HbA1c of $9 \%$ has a 1.54-times greater risk of a heart attack than someone with an $\mathrm{HbA} 1 \mathrm{c}$ level of $6 \%$ (Selvin et al 2004). According to the American Association of Clinical Endocrinologists (AACE) Diabetes Mellitus Clinical Practice Guidelines, a glycemic target of HbA1c of $\leq 6.5 \%$ should be encouraged in diabetic patients (AACE 2007).

\section{Stable CAD - multiple treatment options}

A multifaceted approach is required in the management of angina. It includes lifestyle intervention, medical therapy, and revascularization therapy (percutaneous or surgical). Michalsen et al (2006) randomized 101 patients with established CAD to undergo 1-year intensive lifestyle modification vs printed lifestyle advice. Patients assigned to intensive lifestyle modification were extensively informed about the Mediterranean diet; stress 
management and increased physical activity were strongly recommended. Patients in the printed lifestyle advice group received written lifestyle informations only. At 1 year, the patients who underwent comprehensive lifestyle modification showed significantly reduced anginal attacks (-54\% vs $11 \%$, $\mathrm{p}=0.01$ ) with concomitant dose reductions in $30 \%$ of antiischemic medications $(\mathrm{p}=0.004)$ and improved quality of life (Michalsen et al 2006). Boden et al (2007) reported that optimal medical therapy and aggressive management of multiple treatment targets without initial percutaneous coronary intervention can be implemented safely in most patients with chronic stable angina, even those with objective evidence of ischemia and significant multivessel CAD.

The presence of diabetes in CAD patients doubles the risk of adverse cardiovascular events, and aggressive control of blood pressure is associated with significant reduction in diabetes risk. Antianginal and antihypertensive regimes containing primarily ß-blockers and/or thiazide diuretics alter glucose metabolism and/or insulin sensitivity, with a negative impact on glucose and HbA1c levels in diabetic and prediabetic patients. Therefore, novel drugs like ranolazine with its beneficial metabolic effects are crucial for the growing number of patients with diabetes mellitus (Cooper-DeHoff and Pepine 2006).

\section{New mechanistic approaches to myocardial ischemia}

Most patients have relative intolerances to maximum doses of traditional antianginal agents like $\beta$-blockers, nondihydropyridine (verapamil and diltiazem) calcium channel blockers (CCBs), and nitrates. $\beta$-blockers and many CCBs have similar depressive hemodynamic and electrophysiological effects. Therefore antianginal drugs without these limitations are needed. Advances in understanding of myocardial ischemia have prompted evaluation of a number of new antianginal strategies (Borer et al 2003; Marzilli and Klein 2003; Chaitman et al 2005; Chazov et al 2005; Iona Study Group 2005; Messin et al 2005; Tardif et al 2005; Vicari et al 2005; Walker et al 2006).

Ranolazine (Ranexa ${ }^{\circledR}, \mathrm{CV}$ Therapeutics) $([(+) \mathrm{N}-$ (2, 6-dimethylphenyl)-4(2-hydroxy-3- (2-methoxyphenoxy)-propyl)-1-piperazine acetamide dihydrochloride]) is a piperazine derivative and was approved in January 27, 2006 in the US for use in management of chronic angina. It is available in oral and intravenous forms but is approved only for oral use. Ranolazine is useful in patients who have not achieved an adequate response with other antianginal medications, and is used in combination with amlodipine, ß-blockers, or nitrates. Its therapeutic dose range is 500-1000 mg twice daily with the maximum recommended dose is 1000 mg twice daily. Ranolazine undergoes predominantly hepatic metabolism with $<5 \%$ excreted in the urine unchanged (Chaitman 2006).

\section{Beneficial effects of ranolazine}

Ranolazine acts through the following proposed mechanisms.

\section{Effect on cell membrane}

Voltage-gated sodium $\left(\mathrm{Na}^{+}\right)$channels play a fundamental role in the propagation of action potentials in the myocardium. During the plateau phase of the action potential, a small proportion of $\mathrm{Na}^{+}$channels either do not close, or close and then reopen. This late $\mathrm{Na}^{+}$channel opening permits a sustained $\mathrm{Na}^{+}$current to enter myocytes during systole. During ischemia, this enhanced late intracellular $\mathrm{Na}^{+}$ stimulates $\mathrm{Na}^{+} / \mathrm{Ca}^{2+}$ exchanger causing increased cytosolic calcium $\left(\mathrm{Ca}^{2+}\right)$. Increased $\mathrm{Ca}^{2+}$ in turn affects electrical and contractile dysfunction including high diastolic tension. Sustained contraction of ischemic tissue during diastole increases myocardial oxygen consumption and compresses intramural small vessels, thus reducing nutritive blood flow and exacerbating ischemia. Thus $\mathrm{Na}^{+}$-mediated $\mathrm{Ca}^{2+}$ overload mediates a vicious cycle of ischemia begetting more ischemia. Ranolazine reduces calcium uptake indirectly by inhibiting late $\mathrm{Na}^{+}$channels; thus it preserves ionic homeostasis and reduces ischemia-induced contractile dysfunction. Ranolazine, in contrast to older antianginal medications, works downstream of the ischemic insult, and thus may complement the action of traditional antianginal medications (Belardinelli et al 2006a, b).

Fraser et al (2006) measured intracellular $\mathrm{Na}^{+}$and left ventricular (LV) function simultaneously in isolated perfused rat's hearts. The sea anemone toxin II (ATX-II) (inhibitor of late $\mathrm{Na}^{+}$channel) was added to the recirculation perfusate. It causes increased diastolic $\mathrm{Ca}^{2+}$ and $\mathrm{LV}$ dysfunction. Ranolazine when given after ATX-II exposure reverses its effect on diastolic $\mathrm{Ca}^{2+}$ with improved $\mathrm{LV}$ function.

At the therapeutic concentration, ranolazine inhibits 2 ion channels: late $\mathrm{Na}^{+}$channel and delayed rectifier potassium channel. Inhibition of delayed rectifier potassium channel prolongs ventricular action potential, whereas inhibition of late $\mathrm{Na}^{+}$channel has the opposite effects and shortens the action potential duration (APD). The net effect of ranolazine on APD depends on the relative magnitude of reductions in inward $\mathrm{Na}^{+}$and outward $\mathrm{K}^{+}$currents during repolarization (Scirica et al 2007). 
As opposed to its effect on ventricular myocardial APD, ranolazine does not prolong APD of midmyocardium (M cells) and Purkinjee fibers at any concentrations. This distinguishes it from other agents that block delayed rectifier potassium channel like sotalol, which induces prolongation of APD and thus increases the risk of early afterdepolarizations (EADs), which can precipitate torsade de pointes. In an experimental study by Antzelevitch et al (2004), ranolazine showed a significant reduction in sotalol-induced prolongation of APD and EADs. Ranolazine did not induce torsade de pointes even at high concentrations. Despite the fact that ranolazine lengthens the QT interval, ranolazine-induced prolongation of APD is rate dependent (ie, does not display reverse rate-dependent prolongation of APD) and is not associated with EADs, triggered activity, an increase in spatial dispersion of repolarization, or polymorphic ventricular tachycardia. Thus ranolazine may have an antiarrhythmic property due to its ion channel effects, similar to chronic amiodarone therapy.

Ranolazine exerts its antianginal effects without causing significant bradycardia and/or lowering systemic blood pressure (Pepine and Wolff 1999; Chaitman et al 2004). Ranolazine may thus be well suited for patients with lower blood pressure or heart rate, in whom the upward titration of antianginal medications with important hemodynamic effects may not be well tolerated.

Apart from its role in angina, due to its unique mechanism of action, ranolazine may be useful in heart failure, certain types of cardiac sodium channel mutations, ventricular and supraventricular arrhythmias, left ventricular diastolic dysfunction with preserved systolic function, and intermittent claudication in skeletal muscles. However, further studies are needed to support this notion (Chaitman 2006).

\section{Metabolic action}

Circulating fatty acid levels rise abruptly during stress of acute myocardial ischemia. Compared with carbohydrate oxidation, there is an increased oxygen consumption to phosphorylate a given amount of adenosine triphosphate during fatty acid oxidation, thereby depleting the already low levels of oxygen. Further, these elevated fatty acids directly suppress the myocardial uptake of oxygen-efficient carbohydrate substrates like glucose, lactate, and pyruvate, thereby promoting oxygen-wasting fatty acid oxidation mechanism with the resultant accumulation of lactate, and its deleterious effect. Ranolazine appears to shift adenosine triphosphate production away from the oxygen-wasting fatty acid oxidation in favor of more oxygen-efficient carbohydrate oxidation
(Chaitman et al 2004a). However, this action appears at very high, nontherapeutic blood levels.

\section{Ranolazine clinical trial program in chronic stable angina}

The Monotherapy Assessment of Ranolazine in Stable Angina (MARISA) trial was the first placebo-controlled trial to establish the antianginal and anti-ischemic effects of ranolazine. Ranolazine 500, 1000, or $1500 \mathrm{mg}$ given twice daily was compared with placebo in a 4-week trial in 191 patients with exertional angina. Other antianginals were discontinued during the trial except for nitrates. Ranolazine at $500 \mathrm{mg}$, $1000 \mathrm{mg}$, or $1500 \mathrm{mg}$ improved total exercise duration (by 94, 103, and 116 seconds(s), $\mathrm{p}<0.005$ compared with placebo) and time to angina $[27+9.5 \mathrm{~s}(\mathrm{p}<0.005), 45.9+9.5 \mathrm{~s}, 59.6$ $+9.6 \mathrm{~s}, \mathrm{p}<0.001]$. Of note is that ranolazine had negligible effects on heart rate and blood pressure (Chaitman et al 2004b). Thus it significantly increased exercise performance throughout its dosing interval at all doses studied.

In the Combination Assessment of Ranolazine In Stable Angina (CARISA) trial, 823 patients with symptomatic chronic angina taking standard doses of atenolol $(50 \mathrm{mg})$, amlodipine $(5 \mathrm{mg})$, or diltiazem $(180 \mathrm{mg}$ ) were randomized to receive twice the daily dose of $750 \mathrm{mg}$ or $1000 \mathrm{mg}$ of ranolazine or placebo. The choice of background therapy was at the discretion of the investigator and the doses were fixed throughout the study which might contribute to the results. Ranolazine reduced the mean number of angina attacks per week from $3.3 \pm 0.3$ for placebo to $2.5 \pm 0.2$ for $750 \mathrm{mg}$ $(p=0.006)$ to $2.1 \pm 0.2$ for $1000 \mathrm{mg}(\mathrm{p}<0.001)$ ranolazine. It also significantly reduced nitroglycerine consumption $(\mathrm{p}<0.02)$. Exercise duration and time to onset of angina increased significantly with ranolazine, independent of changes in blood pressure, heart rate, or background therapy, and persisted for 12 weeks (Chaitman et al 2004a).

The Efficacy of Ranolazine in Chronic Angina (ERICA) trial investigated 565 patients with persisting anginal symptoms despite maximum recommended doses of amlodipine $(10 \mathrm{mg}$ ) to receive twice daily $1000 \mathrm{mg}$ of ranolazine or placebo. Ranolazine significantly reduced frequency of angina and nitroglycerine consumption when added to conventional antianginal medication (Stone et al 2006).

Thus it is clear that as monotherapy, ranolazine improves exercise performance in the absence of clinically meaningful pathophysiologic effects, and these studies provide evidence of additional antianginal and anti-ischemic efficacy in patients who remain symptomatic on standard therapies or maximal amlodipine therapy. 


\section{Challenges in selected populations: efficacy of ranolazine Effect of gender}

Angina pectoris is more common in women (47\%) than in men $(26 \%)$ and with a less favorable prognosis in women. Efficacy of ranolazine in improving exercise duration is not statistically different between men and women (Wenger et al 2007).

\section{Effect of age}

As evident from the ERICA trial (Stone et al 2006), the antianginal efficacy of ranolazine is independent of age.

\section{Diabetes mellitus}

In a post-hoc analysis of the CARISA trial by Timmis et al (2006), 189 diabetic patients and 634 nondiabetic patients with chronic angina on background atenolol $(50 \mathrm{mg})$, diltiazem $(180 \mathrm{mg})$, or amlodipine $(5 \mathrm{mg})$ therapy were randomized to placebo, or ranolazine 750 or $1000 \mathrm{mg}$ twice daily for 12 weeks, during which exercise tolerance, angina frequency, nitroglycerine usage, glucose, $\mathrm{HbAlc}$, and lipids were measured. There was no statistically significant difference for the effect of ranolazine between diabetic and nondiabetic patients in number of weekly anginal episodes $(p=0.81)$ and nitroglycerine use $(p=0.063)$. There were no significant differences in treatment effects between diabetic and nondiabetic patients for exercise duration $(p=0.89)$, time to onset of angina ( $p=0.54$ ), or time to $\geq 1 \mathrm{~mm} \mathrm{ST}$-segment depression $(p=0.44)$. There were no significant differences in serum lipid levels and adverse events by treatment effects between diabetic and nondiabetic patients.

Though ranolazine did not appear to significantly lower fasting glucose levels in diabetic patients, there was a statistically significant reduction of $\mathrm{HbAl} \mathrm{c}$ with ranolazine compared with placebo regardless of concomitant insulin and/or oral hypoglycemic therapy [ranolazine 750 and $1000 \mathrm{mg}$ reduced HbA1c compared with placebo by $0.48 \pm 0.18 \%(p=0.008)$ and $0.70 \pm 0.18 \%(\mathrm{p}=0.0002)$, respectively]. The mean baseline HbA1c (\%) was $7.46 \pm 0.21$ in the placebo group, $7.65 \pm 0.20$ in the $750 \mathrm{mg}$ ranolazine group, and $7.92 \pm 0.21$ in the $1000 \mathrm{mg}$ ranolazine group. Ranolazine 750 and 1000 $\mathrm{mg}$ showed significant changes in $\mathrm{HbA} 1 \mathrm{c}$ levels from baseline value $(-0.50 \pm 0.13$ and $-0.72 \pm 0.13$, respectively) compared with placebo $(-0.02 \pm 0.14)$. The percentage $(<7 \%)$ of diabetic patients with $\mathrm{HbAlc}$ was significantly higher in the ranolazine $1000 \mathrm{mg}$ group $(\mathrm{p}=0.004)$. In particular, the decreases in $\mathrm{HbAlc}$ seen in insulin-treated patients were greater than those seen in noninsulin-treated patients. Further, the HbA1c concentrations appeared to remain consistent beyond 12 weeks of ranolazine treatment in the long-term, open-label extension study ( $\sim 2$ years) (Timmis et al 2006).

In the recent Metabolic Efficiency With Ranolazine for Less Ischemia in Non-ST-Elevation Acute Coronary Syndromes - Thrombolysis in Myocardial Infarction (MERLIN-TIMI 36) trial (Morrow et al 2007a), 6,560 patients with nonST-elevation acute coronary syndrome at moderate to high risk of recurrent cardiovascular events were randomized to receive ranolazine vs placebo within 48 hours of onset of ischemic symptoms in addition to standard therapy for acute coronary syndrome. In the prospective validation of the MERLIN-TIMI 36 trial, ranolazine showed a significant reduction in $\mathrm{HbA} 1 \mathrm{c}$ at 4 months compared with placebo (change from baseline $-0.30 \mathrm{vs}-0.04, \mathrm{p}=0.001$ ). Diabetic patients treated with ranolazine showed a significant decline in $\mathrm{HbAlc}$ from $7.5 \%$ to $6.8 \%$ (change from baseline $-0.64, p<0.001)$. Further, diabetic patients were more likely to achieve an $\mathrm{HbA} 1 \mathrm{c}<7 \%$ at 4 months when treated with ranolazine vs placebo $(59 \%$ vs $49 \%, \mathrm{p}<0.001)$. Also, ranolazine-treated diabetic patients showed less worsening of hyperglycemia by 1 year of follow-up $(14.2 \%$ vs $20.6 \%$, $\mathrm{p}<0.001$ ). Interestingly, in diabetes-free patients at baseline (baseline $\mathrm{HbA1c}$ of $<6 \%$ ), ranolazine showed reduced incidence of new increase in $\mathrm{HbAlc} \geq 6 \%$ (95\% CI 0.53, 0.88, $p=0.003$ ) (Morrow et al 2007b).

\section{Possible explanation for beneficial effects in diabetes}

The above-mentioned beneficial effects in diabetics could possibly be due to a ranolazine-induced decrease in anginal symptoms and increased physical activity, with a resultant increase in insulin sensitivity. Ranolazine's effect on $\mathrm{HbA} 1 \mathrm{c}$ levels may be speculated by its action on cell membrane or its metabolic action. However, future studies should be carried out to validate this finding. Other mechanisms through the effects of ranolazine on insulin release or decrease in insulin resistance need to be explored.

\section{Long-term safety and tolerability of ranolazine}

Ranolazine is well tolerated in high-risk CAD patients in the long term. In The Ranolazine Open Label Experience (ROLE) program (Koren et al 2007), 2.8-year mean followup was done for 746 chronic angina patients on ranolazine. The most common adverse effects reported were dizziness (11.8\%) and constipation (10.9\%). These complaints led to discontinuation in $0.9 \%$ and $0.5 \%$ of patients, respectively. 
On ECG, the mean QTc prolongation was $2.4 \mathrm{~ms}(\mathrm{p}<0.001)$ while QTc prolongation $>500$ ms was noted in only $1.2 \%$ of patients. No subjects were withdrawn for QTc prolongation and no torsades de pointes was reported. Further, the presence of diabetes status was not correlated with increased adverse event-related discontinuation of ranolazine (relative risk 1.04, $\mathrm{p}=0.87$ ).

Syncope and postural hypotension can occur with ranolazine, likely due to alpha-adrenergic receptor blockage at very high doses of up to $2000 \mathrm{mg}$. It is avoidable by the usual practice of initiating ranolazine with the lowest available dose and carefully titrating upward based on both efficacy and tolerability (Chaitman et al 2004a).

In the MERLIN-TIMI 36 trial ranolazine was not effective in reducing the rate of the composite of cardiovascular death and myocardial infarction. But there was a $13.9 \%$ $(p=0.03)$ relative reduction in the risk of recurrent ischemia, and a lower increase in other antianginal therapy in patients in the ranolazine group, with no effect on the composite of cardiovascular death or myocardial infarction. Ranolazine did not adversely affect the risk of all-cause death, sudden cardiac death, or symptomatic documented arrhythmia. Thus, the MERLIN-TIMI 36 trial does not support the use of ranolazine in acute coronary syndrome, but adds to previous safety data and provides additional support for ranolazine as antianginal therapy in chronic angina (Morrow et al 2007a).

Of note is that ranolazine-treated patients in the MERLINTIMI 36 trial showed a significant reduced frequency of tachyand bradyarrhythmias detected by Holter recordings during the first 7 days of randomization (Scirica et al 2007).

On ECG, T-wave notching has been observed at very high plasma ranolazine concentrations. Ranolazine is contraindicated in patients with pre-existing QT prolongation, on QT-prolonging medications, or with hepatic impairment. An ECG should be obtained at baseline and follow-up to evaluate effects on QT interval. Small increases in creatinine have been observed $(>0.1 \mathrm{mg} / \mathrm{dL})$ with ranolazine, which appear to be due to inhibition of tubular secretion without any detrimental effect on glomerular function. Patients with severe renal insufficiency should be closely monitored especially if the patient is uptitrated to the maximum dose (Chaitman 2006). This may be of concern especially in diabetics who often have concomitant severe renal insufficiency.

Ranolazine is an alternative in many patients with chronic angina who cannot undergo successful percutaneous or surgical revascularization due to anatomic conditions such as severe distal vessel disease, small-branch vessel disease, or other factors (Berger 2004). Its efficacy in diabetics with diffuse, multivessel, and small vessel disease may be an attractive option, particularly in patients with a favorable metabolic profile. Prospective randomized studies are necessary in diabetics with ischemia/coronary artery disease, to validate the favorable benefits noted in subgroup analysis.

\section{Conclusion}

Chronic angina is a chronic debilitating illness. Ranolazine is a novel antianginal medication recently approved by the FDA for management of refractory angina. It appears to be a safe and effective antianginal in the management of refractory chronic angina with an advantage of decreased angina frequency, increased exercise tolerance, and no deleterious effects on hemodynamics. In addition to its antianginal property, ranolazine might have a favorable effect on $\mathrm{HbA1c}$ levels in diabetic patients.

\section{Disclosures}

Neither author has any conflicts of interest to disclose.

\section{References}

AACE Diabetes Mellitus Clinical Practice Guidelines Task Force. 2007 American Association of Clinical Endocrinologists medical guidelines for clinical practice for the management of diabetes mellitus. Endocr Pract, 13(Suppl 1):1-68.

Adult Treatment Panel III. 2002. Third Report of the National Cholesterol Education Program (NCEP) Expert Panel on Detection, Evaluation, and Treatment of High Blood Cholesterol in Adults (Adult Treatment Panel III) Final Report. Circulation, 106:3143-421.

Antzelevitch C, Belardinelli L, Zygmunt AC, et al. 2004. Electrophysiological effects of ranolazine, a novel antianginal agent with antiarrhythmic properties. Circulation, 110; 904-10.

Belardinelli L, Shryock JC, Fraser H. 2006a. Inhibition of the late sodium current as a potential cardioprotective principle: effects of the late sodium current inhibitor ranolazine. Heart, 92(Suppl IV):iv6-iv14.

Belardinelli L, Shryock JC, Fraser H. 2006b. The mechanism of ranolazine action to reduce ischemia-induced diastolic dysfunction. Eur Heart J Suppl, 8 (Suppl A), A10-A13.

Berger P. 2004. Ranolazine and other antianginal therapies in the era of the drug-eluting stent. JAMA, 291:365-7.

Bhatt AB, Stone PH. 2006. Current strategies for the prevention of angina in patients with stable coronary artery disease. Curr Opin Cardiol, 21:492-502.

Boden WE, O'Rourke RA, Teo KK, et al. 2007. Optimal medical therapy with or without PCI for stable coronary disease. $N$ Engl J Med, 356:1503-16.

Borer JS, Fox K, Jaillon P, et al. 2003. Antianginal and antiischemic effects of ivabradine, an if inhibitor, in stable angina: a randomized, double-blind, multicentered, placebo-controlled trial. Circulation, 107:817-23.

Chaitman BR. 2006. Ranolazine for the treatment of chronic angina and potential use in other cardiovascular conditions. Circulation, 113:2462-72.

Chaitman BR, Ivleva AY, Ujda M, et al. 2005. Antianginal efficacy of omapatrilat in patients with chronic angina pectoris. Am J Cardiol, 96:1283-9.

Chaitman BR, Pepine CJ, Parker JO, et al. 2004a. Effects of ranolazine with atenolol, amlodipine, or diltiazem on exercise tolerance and angina frequency in patients with severe chronic angina: a randomized controlled trial. JAMA, 291:309-16. 
Chaitman BR, Skettino SL, Parker JO, et al. MARISA Investigators. 2004b. Antiischemic effects and long-term survival during ranolazine monotherapy in patients with chronic severe angina. J Am Coll Cardiol, 43:1375-82.

Chazov EI, Lepakchin VK, Zharova EA, et al. 2005. Trimetazidine in Angina Combination Therapy: the TACT study: trimetazidine versus conventional treatment in patients with stable angina pectoris in a randomized, placebo-controlled, multicenter study. Am J Ther, 12:35-42.

Cooper-DeHoff R, Pepine CJ. 2006. Ranolazine is associated with cardiovascular and metabolic improvement: a win-win for patients with diabetes. Eur Heart J, 27:5-6.

Fraser H, Belardinelli L, Wang L, et al. 2006. Ranolazine decreases diastolic calcium accumulation caused by ATX-II or ischemia in rat hearts. J Mol Cell Cardiol, 41:1031-8.

Gibbons RJ, Abrams J, Chatterjee K, et al. ACC/AHA. 2003. 2002 guideline update for the management of patients with chronic stable angina summary article: a report of the American College of Cardiology/ American Heart Association Task Force on practice guidelines (Committee on the Management of Patients With Chronic Stable Angina). J Am Coll Cardiol, 41:159-68.

Holubkov R, Laskey W, Haviland A, et al. 2002. Angina 1 year after percutaneous coronary intervention: a report from the NHLBI Dynamic Registry. Am Heart J, 144:826-33.

Koren MJ, Crager MR, Sweeney M. 2007. 2007. Long-term safety of a novel antianginal agent in patients with severe chronic stable angina. The Ranolazine Open Label Experience (ROLE). J Am Coll Cardiol, 49:1027-34

Marzilli M, Klein W. 2003. Efficacy and tolerability of trimetazidine in stable angina: a meta-analysis of randomized, double-blind, controlled trials. Coron Artery Dis, 14:171-9.

Messin R, Opolski G, Fenyvesi T, et al. 2005. Efficacy and safety of molsidomine once-a-day in patients with stable angina pectoris. Int $J$ Cardiol, 98:79-89.

Michalsen A, Knoblauch NTM, Lehmann N, et al. 2006. Effects of lifestyle modification on the progression of coronary atherosclerosis, autonomic function, and angina - The role of GNB3 C825T polymorphism. Am Heart J, 151:870-7.

Morrow DA, Scirica BM, Karwatowska-Prokopczuk E, et al. 2007a. Effects of ranolazine on recurrent cardiovascular events in patients with non-st-elevation acute coronary syndromes. The MERLIN-TIMI 36 Randomized Trial. JAMA, 297:1775-83.
Morrow DA, Scirica BM, Chaitman BR, et al. 2007b. Effect of ranolazine on hemoglobin A1c in the MERLIN-TIMI 36 randomized controlled trial. Circulation, 116:II_539-II_540. Abstract 2453.

Pepine CJ, Wolff AA. 1999. A controlled trial with a novel anti-ischemic agent, ranolazine, in chronic stable angina pectoris that is responsive to conventional antianginal agents. Ranolazine Study Group. Am J Cardiol, 84:46-50.

Scirica BM, Morrow DA, Hod H, et al. 2007. Effect of ranolazine, an antianginal agent with novel electrophysiological properties, on the incidence of arrhythmias in patients with non-ST-segment-elevation acute coronary syndrome. Results from the Metabolic Efficiency With Ranolazine for Less Ischemia in Non-ST-Elevation Acute Coronary Syndrome-Thrombolysis in Myocardial Infarction 36 (MERLINTIMI 36) Randomized Controlled Trial. Circulation, 116:1647-52.

Selvin E, Marinopoulos S, Berkenblit G, et al. 2004. Meta-analysis: Glycosylated hemoglobin and cardiovascular disease in diabetes mellitus. Ann Intern Med, 141:421-31.

Serruys P, Unger F, Sousa J, et al. 2001. Comparison of coronary-artery bypass surgery and stenting for the treatment of multivessel disease. $N$ Engl J Med, 344:1117-24.

Stone PH, Gratsiansky NA, Blokhin A, et al. ERICA Investigators. 2006. Antianginal Efficacy of Ranolazine When Added to Treatment With Amlodipine. The ERICA (Efficacy of Ranolazine in Chronic Angina) Trial. J Am Coll Cardiol, 48:566-75.

Tardif J-C, Ford I, Tendera M, et al. 2005. Efficacy of ivabradine, a new selective If inhibitor, compared with atenolol in patients with chronic stable angina. Eur Heart J, 26:2529-36.

The IONA Study G. 2005. Determinants of coronary events in patients with stable angina: Results from the Impact of Nicorandil in Angina Study. Am Heart J, 150:689.e1-689.e9.

Timmis AD, Chaitman BR, Crager M. 2006. Effects of ranolazine on exercise tolerance and $\mathrm{HbA} 1 \mathrm{c}$ in patients with chronic angina and diabetes. Eur Heart J, 27:42-8.

Vicari RM, Chaitman B, Keefe D, et al. 2005. Efficacy and safety of fasudil in patients with stable angina: a double-blind, placebo-controlled, phase 2 trial. J Am Coll Cardiol, 46:1803-11.

Walker A, McMurray J, Stewart S, et al. 2006. Economic evaluation of the impact of nicorandil in angina (IONA) trial. Heart, 92:619-24.

Wenger NK, Chaitman B, Vetrovec GW. 2007. Gender comparison of efficacy and safety of ranolazine for chronic angina pectoris in four randomized clinical trials. Am J Cardiol, 99:11-18. 